\title{
Wavelet analysis for Forecasting Prices and Arrivals of Black Pepper in Karnataka, India
}

\author{
R. Hanumanthaiah ${ }^{1 *}$, Abhishek Singh ${ }^{1}$, Santhosha Rathod ${ }^{2}$ and Ranjit Kumar Paul ${ }^{2}$ \\ ${ }^{1}$ Department of Farm Engineering, Institute of agricultural sciences, BHU, Varanasi, India \\ ${ }^{2}$ Indian Agricultural Statistics Research Institute, New Delhi-10001, India \\ *Corresponding author
}

\begin{tabular}{|l|}
\hline Key w or d s \\
ARIMA, \\
Forecasting, \\
MODWT, Wavelet, \\
Frequency domain \\
\hline Article Info \\
\hline $\begin{array}{l}\text { Accepted: } \\
\text { 07 April } 2018 \\
\text { Available Online: } \\
\text { 10 May 2018 }\end{array}$ \\
\hline
\end{tabular}

A B S T R A C T

Accurate forecasting of Prices and Arrivals of Black pepper is vital for planning and policy purposes. An attempt is made here for modeling and forecasting of Prices and Arrivals of Black pepper for Bengaluru and Somwarpet markets time-series data by using the promising nonparametric methodology of Wavelet analysis in frequency domain. Maximal overlap discrete wavelet transform (MODWT) which, unlike discrete wavelet transform (DWT), does not require the number of data points to be a power of two is employed. Haar wavelet filter is used for computing the same in order to analyze the behaviour of timeseries data in terms of different times and scales. Wavelet methodology in frequency domain and Autoregressive integrated moving average (ARIMA) methodologies are applied for different wavelet decomposition levels, at each stage of decomposing we found the best ARIMA models and we compute five month ahead forecasts for hold-out data. Relevant computer programs are developed in SAS, Ver. 9.3 and R, Ver. 2.15.0 software packages. Compare the Forecasting performance of both the Markets using Root mean square prediction error (RMSPE), Mean absolute prediction error (MAPE) and mean absolute error (MAE). Wavelet analysis found to be better model in forecasting prices and Arrivals of Black pepper in Bengaluru Market, the values of RMSE, MAE and MAPE obtained were smaller than those in Somwarpet Market.

\section{Introduction}

Autoregressive integrated moving average (ARIMA) methodology (Box et al., 2007), which is a parametric approach, has virtually dominated analysis of time series data during last several decades. Here role of various explanatory variables enter into the model implicitly through response variable observations at past epochs. However, quite often it is not possible to postulate appropriate parametric form for the underlying phenomenon and, in such cases; "nonparametric" approach is called for. Accordingly in recent years, an extremely powerful methodology of "wavelet analysis" is rapidly emerging (Antoniadis, 1997; Vidakovic, 1999; Percival and Walden, 2000). Although, a number of research papers have been published dealing with various theoretical aspects of wavelets, their application to data is still a difficult task. Wavelet analysis can be studied in two ways: one is in "time domain" and other is in 
"frequency domain". In respect of the former, Fryzlewicz et al., (2003) developed wavelet process model for forecasting nonstationary time series. Sunil kumar and Prajneshu (2004) applied wavelet thresholding approach for modelling and forecasting of monthly meteorological subdivisions rainfall in eastern Uttar Pradesh, India. Sunil Kumar and Prajneshu (2008) carried out modelling and forecasting of marine fish production of India using wavelet thresholding with auto correlated errors. For the later approach, Renaud et al., (2003) developed methodology for prediction of time series data based on multi scaled decomposition. Almasri et al., (2008) proposed a test statistic by using wavelet decompositions to test the significance of trend in a time series data. a difficult problem of testing for linear trend is presence of dependence among residuals because of which tests for trend based on classical ordinary least squares regression become inappropriate. In many situations, error auto covariance function exhibits a slow decay reflecting possible presence of long memory process. Wavelet analysis has been extensively used for such purposes, since it suitably matches the structure of these processes. The auto covariance function of the wavelet transformed series decay hyperbolically fast at rate much faster than the original process. In general, series that are correlated in the time domain become almost uncorrelated in the time domain. Aminghafari and Poggi $(2007,2012)$ used wavelets and kernel smoothing approach for forecasting nonstationary time series. Azad et al., (2008) developed a wavelet based significance test for periodicities in Indian monsoon rainfall. Ghosh et al., (2010) computed size and power of test for testing significance of trend in Indian monsoon rainfall data using discrete wavelet transform (DWT). Paul et al., (2011) applied wavelet methodology for detection of trend in Indian monsoon rainfall and found that there is significant declining trend.

\section{Materials and Methods}

\section{Basics of wavelets}

The term Wavelet is used to refer to a set of basic functions with a very special structure which is the key to its main fundamental properties. Wavelets are fundamental building block functions, analogous to the trigonometric sine and cosine functions. As with a sine or cosine wave, a wavelet function oscillates about zero. This oscillating property makes the function a wave. However, the oscillations for a wavelet damp down to zero, hence the name wavelet. If $w($.$) is a real-$ valued function defined over the real axis ($\infty, \infty)$ and satisfies two basic properties:

The integral of $\psi($.$) is zero:$

$$
\int_{-\infty}^{\infty} \psi(u) d u=0
$$

The square of $\psi($.$) integrates to unity:$

$$
\int_{-\infty}^{\infty} \psi^{2}(u) d u=1
$$

Then the function is $\psi($.$) called as a wave. A$ good description of wavelets can be found in Daubechies (1992), Ogden (1997) and Percival and Walden (2000).

\section{Maximal Overlap Discrete Wavelet Transforms (MODWT)}

The MODWT is a linear filtering operation that transforms a series into coefficients related to variations over a set of scales. It is similar to DWT, in that, both are linear filtering operations producing a set of timedependent wavelet and scaling coefficients. Both have basis vectors associated with a location $\mathrm{t}$ and a unit less scale $\tau_{\mathrm{j}}=2^{\mathrm{j}}-1, \mathrm{~J}_{0}$ for 
each decomposition level $\mathrm{j}=1, . ., \mathrm{J}_{0}$. Both are suitable for the analysis of variance (ANOVA) and for multi resolution analysis (MRA). However, MODWT differs from DWT in the sense that it is highly redundant, nonorthogonal transform (Percival and Walden 2000). It retains down sampled values at each level of the decomposition that would otherwise be discarded by DWT. The MODWT is well defined for all sample sizes $\mathrm{N}$, whereas for a complete decomposition of $\mathbf{J}$ levels, DWT requires $\mathrm{N}$ to be a multiple of $2^{\mathrm{J}}$. MODWT offers several advantages over DWT. Redundancy of MODWT facilitates alignment of the decomposed wavelet and scaling coefficients at each level with original time series, thus enabling a ready comparison between the series and its decomposition. ANOVA derived using MODWT is not influenced by circular shifting of input time series, whereas values derived using DWT depend on starting point of a series (Percival and Walden, 2000). Finally, redundancy of MODWT wavelet coefficients modestly increases effective degrees of freedom on each scale and thus decreases variance of certain wavelet-based statistical estimates. Since MODWT is energy conserving, it is well suited for analyzing scale dependence of variability in ANOVA studies (Percival and Mofjed, 1997).

\section{MODWT filters}

A linear filter $a_{t}$ is a sequence of weights, i.e. $a_{t} \equiv\left\{\ldots a_{-2}, a_{-1}, a_{0}, a_{1}, a_{2}, \ldots\right\}$ Linear filtration of a time-series $X_{t}$ (or a stochastic process $\left\{X_{t}\right\}$ ) is defined as

$$
a_{t} \otimes x_{t} \equiv \sum_{m} a_{m} x_{t-m}
$$

Where ${ }^{\otimes}$ stands for convolution operation. An important characteristic of this filter is its frequency response defined as the Fourier transform of $a_{t}$, i.e.

$$
A(f)=\sum_{t} a_{t} e^{-i 2 \pi f t},-\frac{1}{2}<f<\frac{1}{2}
$$

Where $f$ is the frequency. MODWT may be thought of as linear filtration of the time-series or the stochastic process with a special set of linear filters: Wavelet filter $\widetilde{h}_{x}$ and scaling filter ${\widetilde{g_{I}}}$. These are interconnected via the so called quadrature mirror relationship

$\tilde{g}_{l}=(-1)^{l+1} \tilde{h}_{L-1-l}, \tilde{h}_{l}=(-1)^{l} \tilde{g}_{L-1-l}$ (3.49).

And fulfill the conditions:

$\sum_{l=0}^{L-1} \tilde{h}_{l}=0, \sum_{l=0}^{L-1} \tilde{h}_{l}^{2}=\frac{1}{2}$ And $\sum_{l=-\infty}^{L-1} \tilde{h}_{l} \tilde{h}_{l+2 n}=0$ for all nonzero integers $n . . . . . \ldots \ldots . .(3.50)$

$\sum_{l=0}^{L-1} \tilde{g}_{l}=0, \sum_{l=0}^{L-1} \tilde{g}_{l}^{2}=\frac{1}{2} \quad$ And $\quad \sum_{l=-\infty}^{\infty} \tilde{g}_{l} \tilde{g}_{l+2 n}=0$ for all nonzero integers $n$.......... (3.51)

Let $\tilde{h}_{j, l}$ and $\tilde{g}_{j, l}$ be respectively $\mathrm{j}^{\text {th }}$ level MODWT wavelet and scaling filters and let $\mathrm{L}_{\mathrm{j}}$ be width of $\mathrm{j}^{\text {th }}$ level equivalent wavelet and scaling filter. Thus, $\tilde{h}_{j, l}=\tilde{g}_{j, l}=0$ for $1<0$ and $1 \geq \mathrm{L}_{\mathrm{j} .}$ Let $\tilde{H}_{j}(f)$ and $\left|\tilde{G}_{j}(f)\right|_{\text {be respectively }}$ frequency responses of $\tilde{h}_{j, l}$ and ${ }^{\tilde{g}_{j, l}}$ Then

$\left|\tilde{H}_{j}(f)\right| \approx\left\{\begin{array}{l}1, \frac{1}{2^{J+1}}<f \leq \frac{1}{2^{j}} \\ 0, \text { otherwise }\end{array}\right.$

$$
\left|\widetilde{G}_{j}(f)\right| \approx\left\{\begin{array}{l}
1,0<f \leq \frac{1}{2^{j+1}} \\
0, \text { otherwise }
\end{array}\right.
$$

Thus, $\tilde{h}_{j, l}$ is a band pass filter for range of 
frequencies $\frac{1}{2^{J+1}}<f \leq \frac{1}{2^{j}}$ and $\tilde{g}_{j, l}$ is a low pass filter for range of frequencies $0<f \leq \frac{1}{2^{j+1}}$, For Haar wavelet filters

$$
\tilde{h}_{j, l} \equiv\left\{\begin{array}{l}
\frac{1}{2^{j}}, l=0, \ldots \ldots .2^{j-1}-1 \\
-\frac{1}{2^{j}}, l=2^{j-1}, \ldots . . .2^{j-1} \\
0, \text { otherwise }
\end{array}\right.
$$

And for Haar scaling filters:

$$
\tilde{g}_{j, l} \equiv\left\{\begin{array}{l}
\frac{1}{2^{j}}, l=0, \ldots . . .2^{j-1} \\
0, \text { otherwise }
\end{array}\right.
$$$$
\tilde{h}_{j, l} \quad \tilde{g}_{j, l}
$$

Lengths of Haar filters and $\quad$ are $\mathrm{L}_{\mathrm{j}}=2^{\mathrm{j}}$.

\section{MODWT coefficients}

For a redundant transform, like MODWT, an $\mathrm{N}$ sample input time-series will have an $\mathrm{N}$ sample resolution level.

Therefore, features of wavelet coefficients in a multiresolution analysis (MRA) will be lined up with original time-series in a meaningful way. For a time-series $\mathrm{X}$ with arbitrary sample size $\mathrm{N}$, the $\mathrm{j}^{\text {th }}$ level MODWT wavelet $\left(\widetilde{W}_{i}\right)$ and scaling $\left(\widetilde{V}_{i}\right)$ coefficients are defined as:

$$
\begin{gathered}
\tilde{w}_{j, t} \equiv \sum_{l=0}^{L_{j-1}} \tilde{h}_{j, l} X_{t-1 \bmod N} \\
\tilde{V}_{j, t} \equiv \sum_{l=0}^{L_{j-1}} \tilde{g}_{j, l} X_{t-1 \bmod N}
\end{gathered}
$$

Where $\tilde{h}_{j, l} \equiv \frac{h_{j, l}}{2^{j / 2}}$ are $\mathrm{j}^{\text {th }}$ level MODWT wavelet filters, and $\tilde{g}_{j, l} \equiv \frac{g_{j, l}}{2^{j / 2}}$ are $\mathrm{j}^{\text {th }}$ level MODWT scaling filters, $L_{j}$ is width of $j^{\text {th }}$ level equivalent wavelet and scaling filters. For a time-series $\mathrm{X}$ with $\mathrm{N}$ samples, MODWT yields and additive decomposition or MRA given by

$$
X \equiv \sum_{j=1}^{J_{0}} \widetilde{D}_{j}+\tilde{S}_{j_{0}}
$$

Where,

$$
\begin{gathered}
\tilde{D}_{j, t} \equiv \sum_{l=0}^{N-1} \tilde{u}_{j, l} \tilde{w}_{j, l+1 \bmod N} \\
\tilde{S}_{j, t} \equiv \sum_{l=0}^{N-1} \tilde{v}_{j, l} \tilde{v}_{j, l+1 \bmod N}
\end{gathered}
$$

$\tilde{u}_{j, l}$ and $\tilde{v}_{j, l}$ being the filters obtained by periodizing $\tilde{h}_{j, l}$ and $\tilde{g}_{j, l}$ According to eq. (3.58), at a scale $\mathrm{j}$, a set of coefficients $\left\{\mathrm{D}_{\mathrm{j}}\right\}$ each with the same number of samples $(\mathrm{N})$ as in the original signal $(\mathrm{X})$ is obtained. These are called wavelet "details" and capture local fluctuations over whole period of a time-series at each scale. Set of values $\widetilde{S}_{j_{0}}$ provide a "smooth" or overall "trend" of the signal and adding $\mathrm{D}_{\mathrm{j}}$ to ${\widetilde{j_{0}}}$, for $\mathrm{j}=1,2 \ldots, \mathrm{J}_{0}$, gives an increasingly more accurate approximation for it. This additive form of reconstruction allows prediction of each wavelet subseries $\left(\mathrm{D}_{\mathrm{j}}, \widetilde{S}_{j_{0}}\right)$ separately and adding individual predictions an aggregate forecast is generated.

\section{Choosing number of levels}

A time-series can be completely or partially decomposed into a number of levels. For complete decomposition of a series of length 
$\mathrm{N}=2^{\mathrm{J}}$ using DWT, maximum numbers of levels in the decomposition is $\mathrm{J}_{0} \leq \mathrm{J}$ suffices for many applications. A $\mathrm{J}_{0}$ level DWT decomposition requires that $\mathrm{N}$ be an integral multiple of $2^{J_{0}}$.

The MODWT can accommodate any sample size $\mathrm{N}$ and, in theory, any $\mathrm{J}_{0}$. In practice, largest level is commonly selected such that $\mathbf{J}_{0}$ $\leq \log _{2}(\mathrm{~N})$ in order to preclude decomposition at scales longer than total length of the timeseries.

In particular, for alignment of wavelet coefficients with the original series, condition $L_{j_{0}}<\mathrm{N}$ (i.e. width of equivalent filter at $J_{0}{ }^{\text {th }}$ level is less than sample size) would be satisfied to prevent multiple wrappings of the time-series at level $\mathbf{J}_{0}$. Selection of $\mathbf{J}_{0}$ determines the number of octave bands and thus numbers of scales of resolutions in the decomposition.

\section{Forecasting through wavelet methodology}

Following Renaud et al., (2003), Azad et al., (2008), Aminghafari and Poggi (2007) and Aminghafari and Poggi (2012) for the Haar wavelet, reconstruction formula for $\mathrm{t}=\mathrm{N}+1$ can be written as

$$
X_{N+1}=\widetilde{V}_{J, N+1}+\sum_{j=1}^{J} \widetilde{w}_{J, N+1}
$$

Then, to predict $X_{N+1}$, it suffices to predict MODWT approximation and detail coefficients $\tilde{V}_{J, N+1}$ and $\tilde{w}_{J, N+1}$. Hence, idea is to predict for each scale unknown MODWT coefficients by a linear combination of their past values dydically lagged starting from $\mathrm{N}$ :

$$
\widetilde{w}_{J, N+1}=\sum_{k=1}^{A_{j}} a_{j, k} \widetilde{w}_{j, N-2^{j}(k-1)}
$$

$\tilde{\widetilde{v}}_{J, N+1}=\sum_{k=1}^{A_{j+1}} a_{j+1, k} \tilde{V}_{j, N-2^{j}(k-1)}$

It may be noted that the past values appearing on the right hand sides of above equations must depend only on the past observations of the process itself. The explanatory variables are selected using dyadically lagged values of MODWT coefficients of nonredundant (i.e. decimated) wavelet transform corresponding to dyadic grid adapted to (i.e. ending at) last observed value. Then, complete prediction equation of $X_{N+1}$, when $N$ observations $X_{1}$, $\mathrm{X}_{2} \ldots \mathrm{X}_{\mathrm{N}}$ are given, is of the following form:

$\hat{X}_{N+1}=\sum_{j=1}^{J} \sum_{k=1}^{A_{j}} a_{j, k} \tilde{\tilde{w}}_{j, N-2^{j}(k-1)}+\sum_{k=1}^{A_{J+1}} a_{j+1, k} \tilde{\tilde{v}}_{j, N-2^{j}(k-1)}$

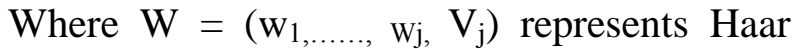
MODWT of $\mathrm{X} \quad\left(X=\tilde{v}_{J}+\sum_{j=1}^{J} \tilde{w}_{j}\right)$ For example choosing $A_{j}=1$ for all resolution levels $\mathrm{j}$, leads to the prediction

$$
\hat{X}_{N+1}=a j+\widetilde{\widetilde{w}}_{j, N}+a_{j+1} \tilde{\widetilde{v}}_{j, N}
$$

\section{Results and Discussion}

Since the wavelet-based forecasting procedure works best for large sample sizes around 100 and larger, so we take a large amount of Monthly data of Prices and Arrivals of Black Pepper. The data is collected from KSAMB (Karnataka State Agricultural Marketing Board, Yeshwanthpur) and www.indiastat.com over a period of January 2003 to October 2017. Both data series are divided in two sets: training set and validation set. First, the model is fitted using the training data set and then forecast the fitted model over the validation period. For the training set data, we take values from January 2003 to May 2017 and predict next five month values (from June 2017 to October 2017). 
For computation of MODWT and forecasting of prices and arrivals of Black Pepper in Bengaluru and Somwarpet markets by Wavelet approach, we followed methodology described in materials and methods section by Paul et al., (2013).

Here, we take $J_{0}$ as 5 . Haar wavelet is used for analyzing the data on a scale by scale basis to reveal it localized nature as exhibited by MODWT coefficients at level 5 for both prices and Arrivals in Bengaluru and Somwarpet markets separately. The coefficients at the top are "high-frequency" and below "low frequency" information. The wavelet coefficients do not remain constant over time and reflects the changes of the data at various time-epochs.

The locations of abrupt jumps can be spotted by looking for vertical (between levels) clustering of relatively large coefficients. From the wavelet coefficients plotted above, the original function can be reconstructed by using Maximum Overlap Discrete Wavelet Transform (MODWT). Here, $X$ denotes original time series plot, $\mathrm{W}_{1}$ to $\mathrm{W}_{5}$ denote the wavelet details components, and $\mathrm{V}_{5}$ denotes the smoothed component of MODWT. A perusal indicates that localized variation in the data is detected at lower scale, whereas global variation is detected at higher scale. The wavelet coefficients are related to differences (of various orders) of (weighted) average values of portions of $\mathrm{X}_{\mathrm{t}}$ concentrated in time.

The Wavelet procedure consists of the following steps:

At the first stage, we decompose the original time series with wavelet using $\mathrm{R}$ software. As the discrete wavelet transform is very effective for time series, we use it for data decomposition. This transform decomposes the data into coarse and finer parts. The coarse scales exhibit the trend while finer scales show the seasonal influences and noise. We use Haar wavelet with decomposition level 5.

So we have

$f(t)=X+W_{1}+W_{2}+W_{3}+W_{4}+W_{5}+V_{5}$.

After the decomposition, appropriate ARIMA model is fitted on each scale and model is used to forecast the future values for the one year for each part.

Then using these decomposed and extended signals on different scales we reconstruct the Signal with the help of following equation. Here $f^{e x t}(t)$ is the estimate price for next one year ahead

$f^{e x t}(t)=X^{e x t}+W_{1}^{e x t}+W_{2}^{e x t}+W_{3}^{e x t}+W_{4}^{e x t}+W_{5}^{e x t}+V_{5}^{e x t}$

A Feed forward forecasting model by wavelet approach was fitted to the data with the help of ' $R$ ' program for each prices and arrivals time series of Black Pepper for Bengaluru and Somwarpet market, which are graphically represented in Figure 1, 2, 3 and 4.

The performance of prediction is evaluated by the most used statistical measures of error: Mean Absolute Error (MAE), Root Mean Square Error (RMSE) and Mean Absolute Percentage Error (MAPE). These are defined as

$R M S E=\sqrt{\sum_{t=1}^{n}\left(y_{t}-\hat{y}_{t}\right)^{2} / n} * 100$

$M A E=\frac{1}{n} \sum_{t=1}^{n}\left|y_{t}-\hat{y}_{t}\right|$

$M A P E=\frac{1}{n} \sum_{t=1}^{n}\left|\frac{y_{t}-\hat{y}_{t}}{y_{t}}\right|$ 
Fig.1 MODWT of black pepper prices of Bengaluru market

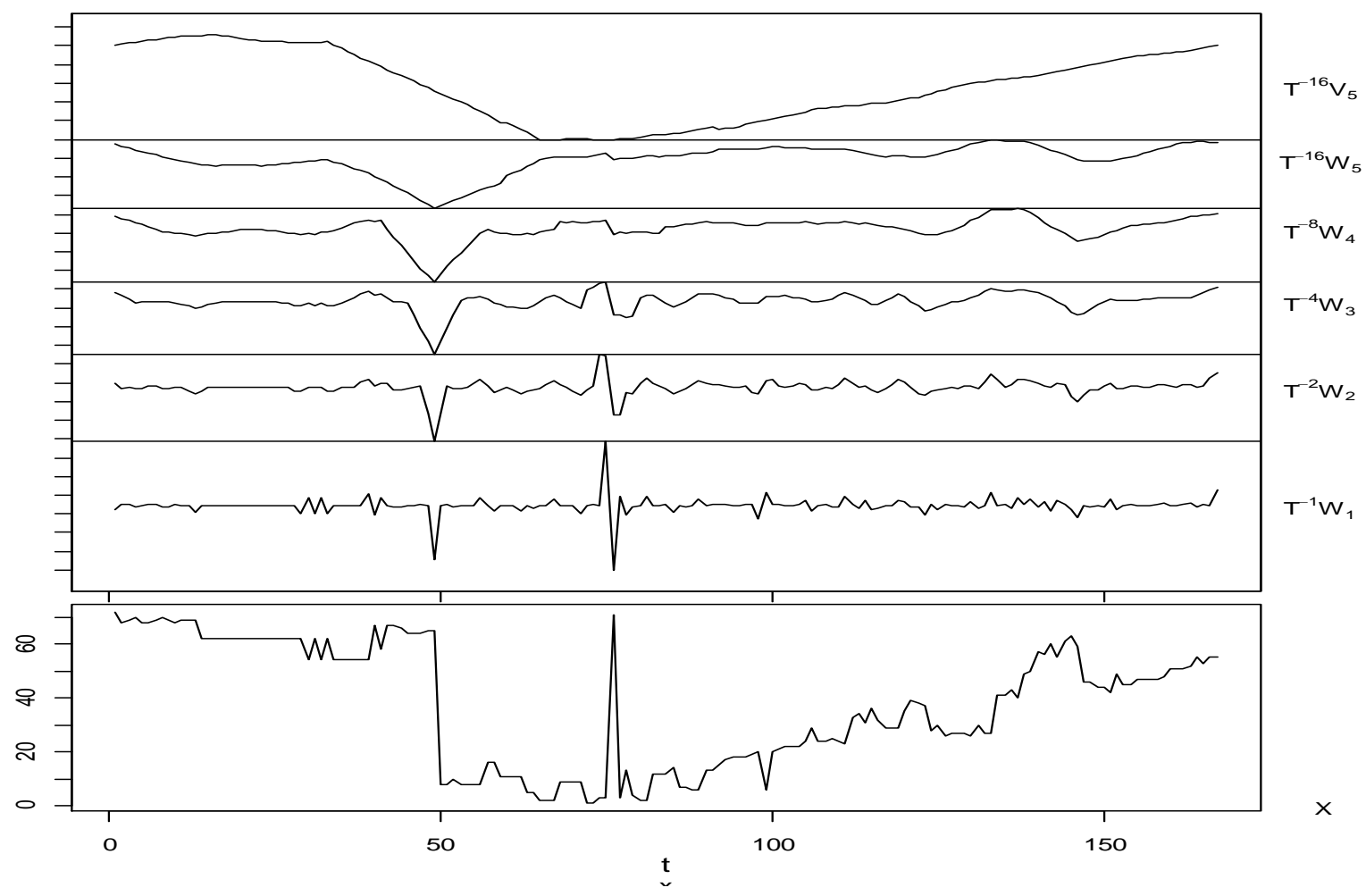

Fig.2 MODWT of black pepper arrivals of Bengaluru market

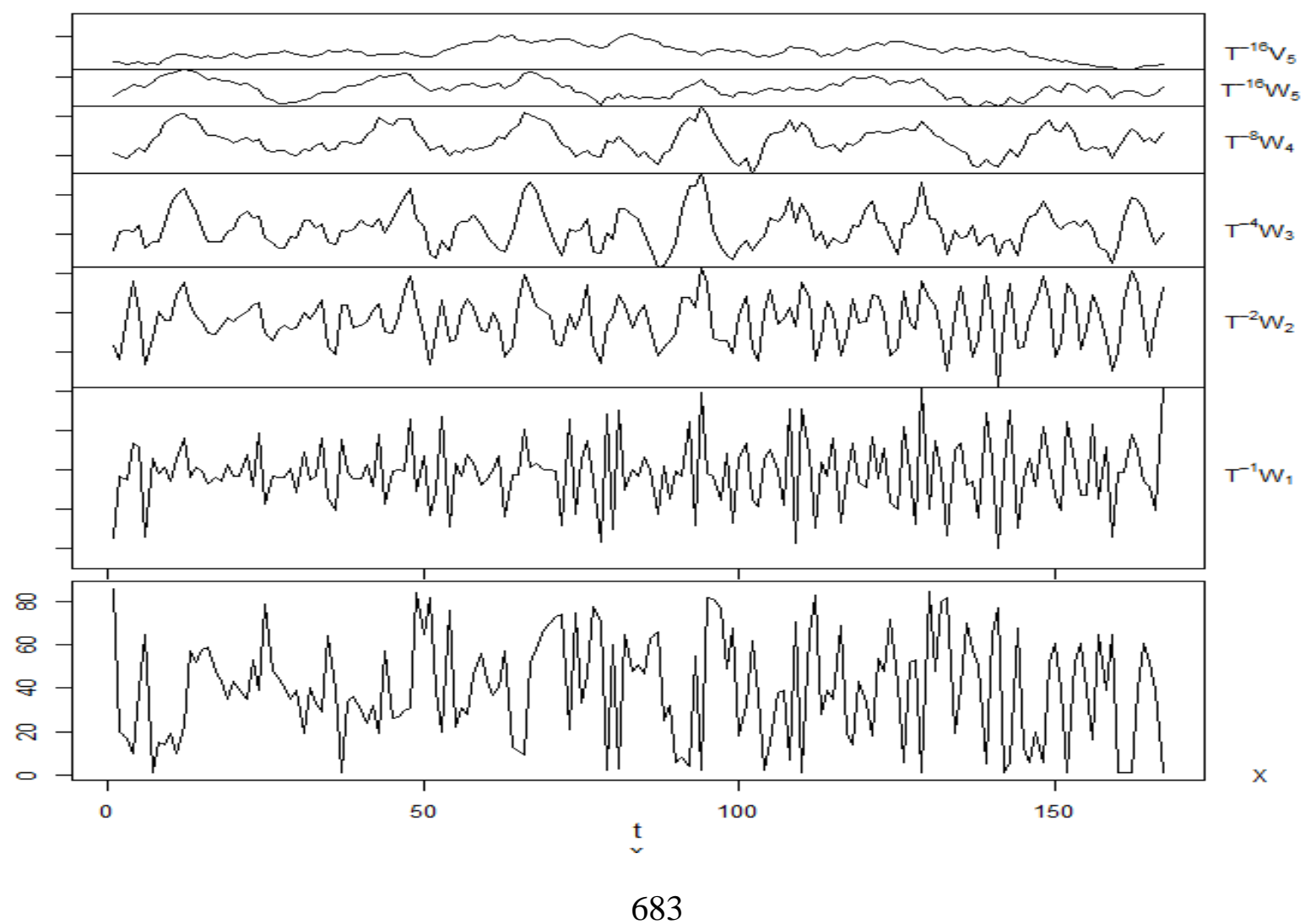


Fig.3 MODWT of black pepper prices of Somwarpet market

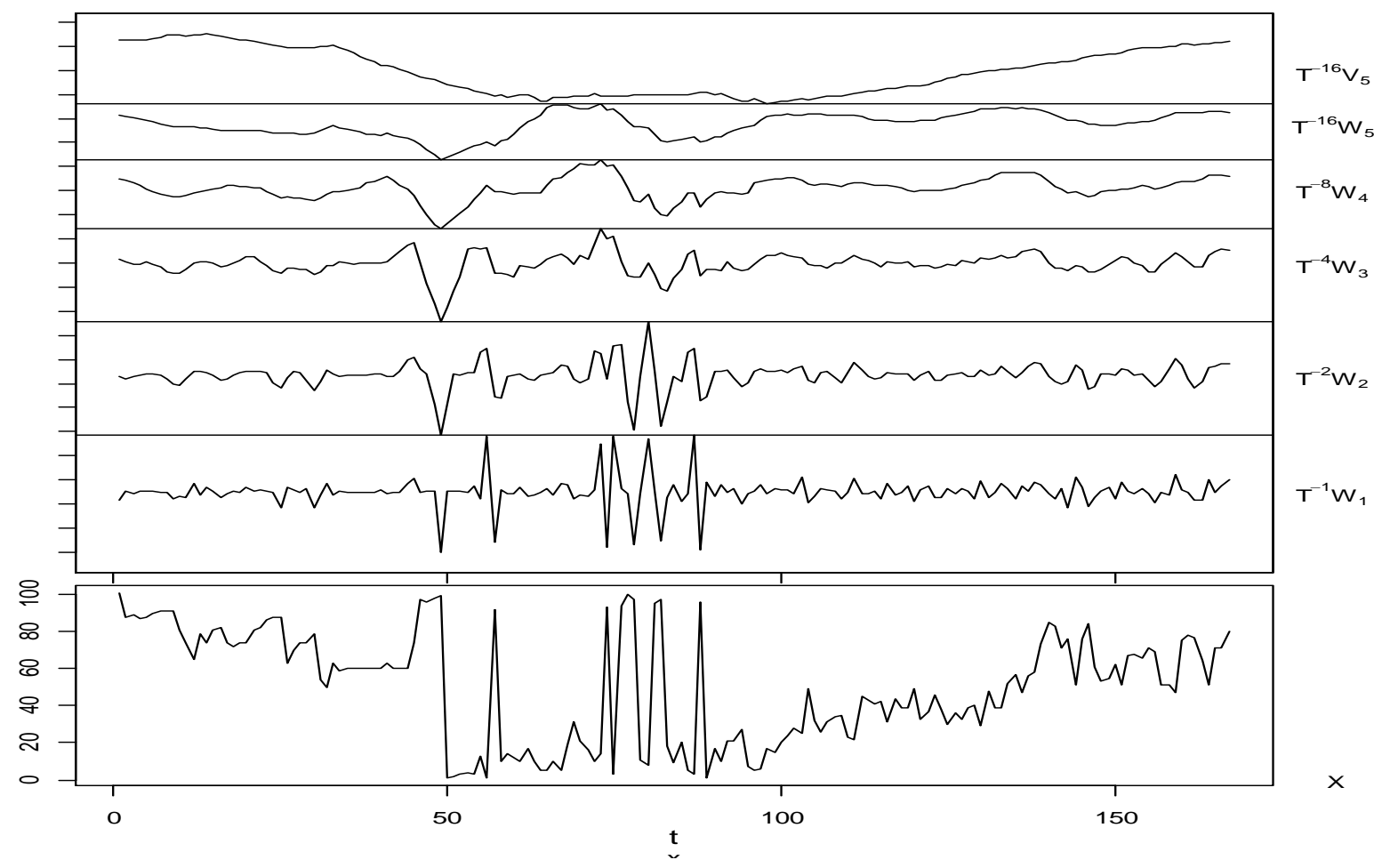

Fig.4 MODWT of black pepper arrivals of Somwarpet market

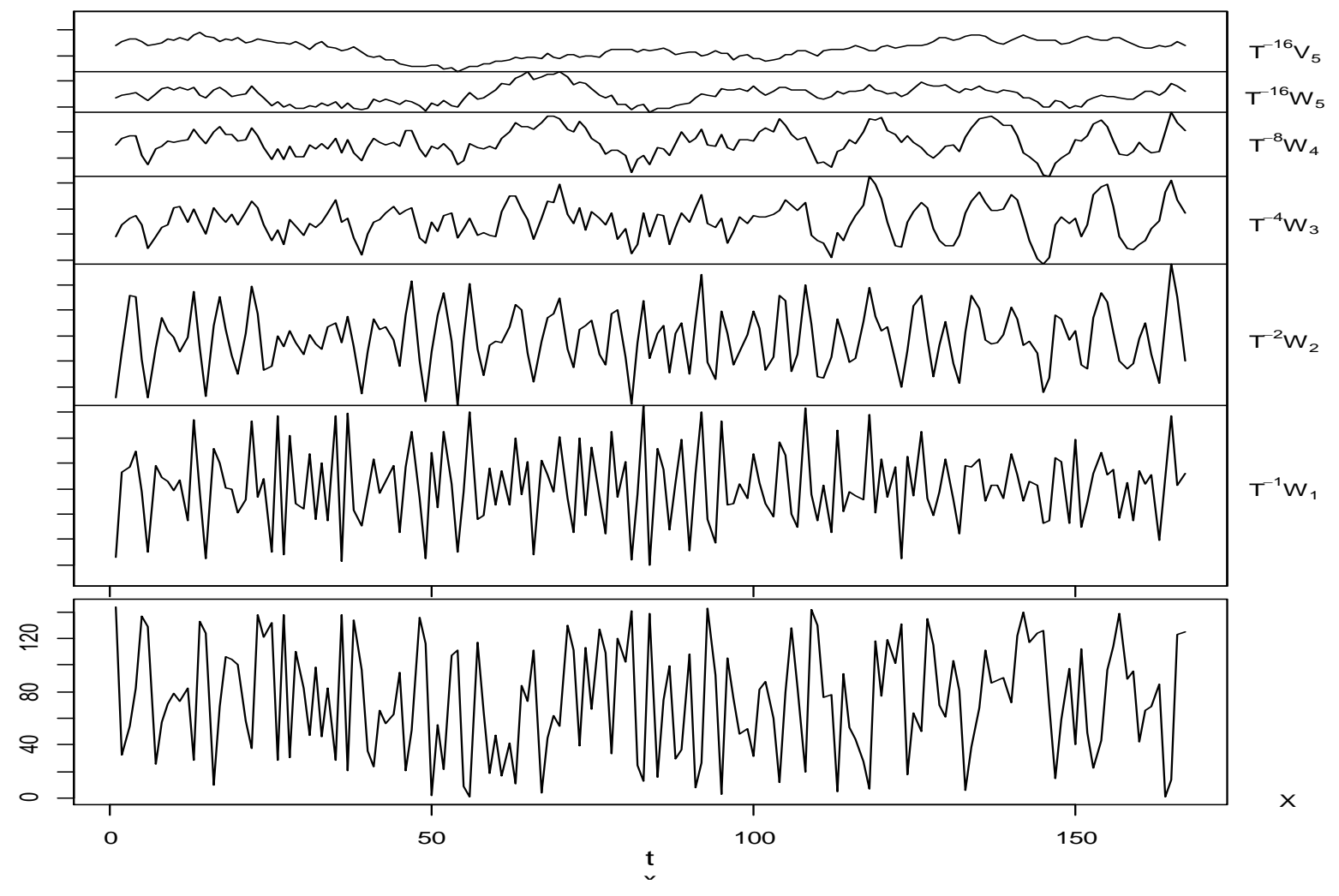


Table.1 Accuracy measures of training data of Bengaluru and Somwarpet markets

\begin{tabular}{|c|c|c|c|c|}
\hline \multirow{2}{*}{$\begin{array}{c}\text { Accuracy } \\
\text { Statistics }\end{array}$} & \multicolumn{2}{|c|}{ Bengaluru Market } & \multicolumn{2}{c|}{ Somwarpet Market } \\
\hline MAE & Prices & Arrivals & Prices & Arrivals \\
\hline RMSE & 7570.421 & 55.405 & 8619.739 & 609.819 \\
\hline MAPE & 11346.395 & 80.843 & 14138.788 & 1130.097 \\
\hline
\end{tabular}

Table.2 Accuracy measures of testing data of Bengaluru and Somwarpet markets

\begin{tabular}{|c|}
\hline $\begin{array}{c}\text { Accuracy } \\
\text { Statistics }\end{array}$ \\
\hline MAE \\
\hline RMSE \\
\hline MAPE \\
\hline
\end{tabular}

\begin{tabular}{|c|c|c|c|}
\hline \multicolumn{2}{|c|}{ Bengaluru Market } & \multicolumn{2}{|c|}{ Somwarpet Market } \\
\hline Prices & Arrivals & Prices & Arrivals \\
\hline 10224.53 & 36.328 & 16117.005 & 679.787 \\
\hline 11599.58 & 54.147 & 19213.402 & 794.256 \\
\hline 17.759 & 93.354 & 31.331 & 87.277 \\
\hline
\end{tabular}

Table.3 Actual vs. Predicted values for Bengaluru market

\begin{tabular}{|c|c|c|c|c|}
\hline \multirow{2}{*}{ Month } & \multicolumn{2}{|c|}{ Prices } & \multicolumn{2}{c|}{ Arrivals } \\
\hline & Actual & Predicted & Actual & Predicted \\
\hline Jun-17 & 57000 & 63073.25 & 20 & 35.9592 \\
\hline Jul-17 & 53000 & 64219.98 & 50 & 36.49445 \\
\hline Aug-17 & 51500 & 65327.06 & 120 & 36.79548 \\
\hline Sep-17 & 50000 & 66369.23 & 180 & 37.03347 \\
\hline Oct-17 & 48500 & 67323.61 & 110 & 37.13924 \\
\hline Accuracy & RMSE & 11599.58 & RMSE & 54.14 \\
\hline Statistics & MAE & 10224.53 & MAE & 36.32 \\
\hline
\end{tabular}

Table.4 Actual vs. Predicted values for Somwarpet market

\begin{tabular}{|c|c|c|c|c|}
\hline \multirow{2}{*}{ Month } & \multicolumn{3}{|c|}{ Prices } & \multicolumn{2}{c|}{ Arrivals } \\
\cline { 2 - 5 } & Actual & Predicted & Actual & Predicted \\
\hline Jun-17 & 48894 & 75020.84 & 910 & 76.36469 \\
\hline Jul-17 & 46000 & 75027.86 & 1000 & 78.41139 \\
\hline Aug-17 & 49890 & 75147.09 & 570 & 77.82614 \\
\hline Sep-17 & 46800 & 75394.34 & 640 & 78.05489 \\
\hline Oct-17 & 62000 & 75773.52 & 490 & 79.58356 \\
\hline Accuracy & RMSE & 19213.40 & RMSE & 794.25 \\
\hline Statistics & MAE & 16117.00 & MAE & 679.78 \\
\hline
\end{tabular}


Table.5 Wavelet ARIMA models

\begin{tabular}{|c|c|c|c|c|}
\hline $\begin{array}{c}\text { Decomposition } \\
\text { levels }\end{array}$ & $\begin{array}{c}\text { Bengaluru } \\
\text { Prices }\end{array}$ & $\begin{array}{c}\text { Bengaluru } \\
\text { Arrivals }\end{array}$ & $\begin{array}{c}\text { Somwarpet } \\
\text { Prices }\end{array}$ & $\begin{array}{c}\text { Somwarpet } \\
\text { Arrivals }\end{array}$ \\
\hline W1 & $(0,0,1)$ & $(1,0,0)$ & $(0,0,1)$ & $(2,0,0)$ \\
\hline W2 & $(1,0,1)$ & $(1,0,1)$ & $(1,0,2)$ & $(1,0,1)$ \\
\hline W3 & $(1,0,1)$ & $(1,0,1)$ & $(1,0,2)$ & $(1,0,1)$ \\
\hline W4 & $(2,0,1)$ & $(2,0,1)$ & $(2,0,2)$ & $(2,0,2)$ \\
\hline W5 & $(1,1,0)$ & $(1,1,0)$ & $(1,1,1)$ & $(1,1,1)$ \\
\hline V5 & $(1,1,1)$ & $(1,1,1)$ & $(1,1,1)$ & $(1,1,1)$ \\
\hline
\end{tabular}

Where ${ }^{y_{t}}$ are the actual price (arrivals) and $\hat{y}_{t}$ forecasted price (arrivals) respectively and $\mathrm{n}$ is total number of observations. The results of the Accuracy measures for Bengaluru and Somwarpet Market are given in the Table 1 and 2 respectively.

Table 1 Results of Accuracy measures for training data revealed that all the measures of accuracy are low for both Prices and Arrivals in Bengaluru market compared to Somwarpet Market.

Table 2 Results of Accuracy measures for testing data revealed that all the measures of accuracy are low except MAPE for both Prices and Arrivals in Bengaluru market compared to Somwarpet Market.

Forecasting of prices and arrivals of black pepper for Bengaluru and Somwarpet markets

Five month ahead Forecasts of monthly Prices and Arrivals for the Bengaluru and Somwarpet Markets are done from June 2017 to October 2017(five month) and the results are depicted in Tables 3 and 4

Results of Tables 3 and 4 reveals that forecasted prices of Bengaluru Market are very near to actual values when we compared with Somwarpet Market, where as in case of Somwarpet Market Arrivals, fitted values are not near to actual values; by this we can conclude that wavelet Analysis comparatively gives good results for Bengaluru Market Prices and Somwarpet Arrivals.

Results in Table 5 indicates that ARIMA models are fitted at different decomposition levels and we conclude that in the V5 level (Smoothed MODWT) for both the Markets Prices and Arrivals ARIMA $(1,1,1)$ found to be best models for forecasting prices and Arrivals.

Wavelet analysis in frequency domain employing Haar wavelet function is used for forecasting prices and arrivals of Black pepper for Bengaluru and Somwarpet Market. From the results it is found to be better model in predicting the Prices and Arrivals of Bengaluru and Somwarpet market respectively as the values of Accuracy statistics i.e. RMSE, MAPE and MAE are low, whereas this wavelet decomposition is not suitable for prices and arrivals of Somwarpet and Bengaluru markets respectively from the values of accuracy statistics are high. Extension of Wavelet and ARIMA with different wavelets like Daubechies, Morlet could be tried in due course of time.

\section{References}

Almasri, A., Locking, H. and Shukur, G., 2008. Testing far climate warming in 
Sweden during 1850-1999, using wavelets analysis. Journal of Applied Statistics, 35, 431-43.

Aminghafari, M and Poggi, J.M., 2007. Forecasting Time series using Wavelets. International Journal of Wavelets Multiresolution Information Processing, 5:709-724.

Antoniadis, A., 1997. Wavelets in Statistics. A Review, Journal of Italian statistical society, 6:97-144.

Azad, S., R. Narasimha, and S. K. Sett, 2008. A Wavelet Based Significance Test for Periodicities in Indian Monsoon Rainfall. International Journal of Wavelets Multiresolution Information Processing, 06: 291.

Daubechies, L.1992. Ten Lectures on Wavelets. SIAM, Philadelphia.

Fryzlewicz, P., Bellegem, S.V and Von Sachs, R., 2003. Forecasting Non stationary Time series by Wavelet process Modelling. Annals of the Institute of Statistical Mathematics, 55, 737-764.

Ghosh, H., Paul, R. K and Prajneshu, 2010. Wavelet Frequency Domain Approach for Statistical Modeling of Rainfall
Time-Series Data. Journal of Statistical Theory and Practice, 4(4).

Ogden, T., 1997. Essential Wavelets for Statistical Applications and Data Analysis. Birkhauser, Boston.

Paul, R.K., Ghosh, H. and Prajneshu, 2011. Wavelet methodology for estimation of trend in Indian monsoon rainfall timeseries data. The Indian Journal of Agricultural Sciences, 81(3).

Percival, D.B and Walden, A.T., 2000. Wavelet Methods for Time Series Analysis. Cambridge University Press, UK.

Renaud, O., J.L. Stark and F.Murtagh, 2003. Prediction based on a multiscale decomposition. International Journal of Wavelets Multiresolution Information Processing, 1: 217-232.

Sunilkumar, G and Prajneshu, 2004. Modelling and forecasting meteorological subdivisions rainfall data using wavelet thresholding approach. Cal. Stat. Assn. Bull, 55: 253265.

Vidakovic, B., 1999. Statistical Modeling by Wavelets. John Wiley, New York.

\section{How to cite this article:}

Hanumanthaiah, R., Abhishek Singh, Santhosha Rathod and Ranjit Kumar Paul. 2018. Wavelet analysis for Forecasting Prices and Arrivals of Black Pepper in Karnataka, India. Int.J.Curr.Microbiol.App.Sci. 7(05): 677-687. doi: https://doi.org/10.20546/ijcmas.2018.705.082 